\title{
Prediction of breast cancer prognosis using gene set statistics provides signature stability and biological context
}

\author{
Gad Abraham 1,2, Adam Kowalczyk2, Sherene Loi3,4, Izhak Haviv5,6,7 and Justin Zobel*1,2
}

\begin{abstract}
Background: Different microarray studies have compiled gene lists for predicting outcomes of a range of treatments and diseases. These have produced gene lists that have little overlap, indicating that the results from any one study are unstable. It has been suggested that the underlying pathways are essentially identical, and that the expression of gene sets, rather than that of individual genes, may be more informative with respect to prognosis and understanding of the underlying biological process.
\end{abstract}

Results: We sought to examine the stability of prognostic signatures based on gene sets rather than individual genes. We classified breast cancer cases from five microarray studies according to the risk of metastasis, using features derived from predefined gene sets. The expression levels of genes in the sets are aggregated, using what we call a set statistic. The resulting prognostic gene sets were as predictive as the lists of individual genes, but displayed more consistent rankings via bootstrap replications within datasets, produced more stable classifiers across different datasets, and are potentially more interpretable in the biological context since they examine gene expression in the context of their neighbouring genes in the pathway. In addition, we performed this analysis in each breast cancer molecular subtype, based on ER/HER2 status. The prognostic gene sets found in each subtype were consistent with the biology based on previous analysis of individual genes.

Conclusions: To date, most analyses of gene expression data have focused at the level of the individual genes. We show that a complementary approach of examining the data using predefined gene sets can reduce the noise and could provide increased insight into the underlying biological pathways.

\section{Background}

Much attention has been given to predicting patient survival from microarray data. In breast cancer, van ' $t$ Veer et al. $[1,2]$ set out to find genes that could be used to predict whether breast cancer patients would experience a metastasis five years after surgery (a binary variable). Their list of 70 genes (NKI70) performed well in predicting the clinical outcome (area under receiver-operating characteristic curve, $\mathrm{AUC} \approx 0.7)$ and is currently commercially available as a prognostic test for breast cancer patients. However, Ein-Dor et al. [3] used the stratified bootstrap to show that many other gene lists of similar predictive ability could be found from the same data.

*Correspondence: jz@csse.unimelb.edu.au

1 Department of Computer Science and Software Engineering, The University of Melbourne, Parkville 3010, VIC, Australia

Full list of author information is available at the end of the article
Moreover, the overlap between the gene lists was low. Similarly, Michiels et al. [4] reported that gene lists derived from seven published cancer studies were highly unstable, and suggested random resampling for validation of such signatures. Many other studies of predicting breast cancer survival using gene expression have followed, with differing gene lists [5]. Reyal et al. [6] examined the performance of nine gene signatures on seven breast cancer datasets. Although the signatures had similar predictive ability, they showed little agreement on the individual predictions -- less than $50 \%$ of the individual predictions agreed -- and had only a small number of overlapping genes.

These results raise several questions. First, are these genes truly associated with cancer and metastasis, or are they spurious, the result of complex models overfitting the data? Second, if these genes are associated with can- 
cer, are they also causally related to it? (a gene may be "down stream" of a cancer-causing gene and therefore be associated with but not cause cancer). Third, can a stable gene list be found at all? Fourth, do the different lists actually represent the same underlying pathways [7] and hence are in more agreement than is otherwise apparent? Interestingly, it is not clear whether there is any advantage in using gene lists, as opposed to single genes, at least from a prediction point of view -- Haibe-Kains et al. [8] found that classifiers built on single genes were as accurate (in terms of AUC) as classifiers based on multiple genes. Similarly, Lai et al. [9] reported that simpler models (univariable models) were more stable than multivariable models, possibly due to overfitting of the latter.

If the different predictive genes truly represent the same underlying biology, then perhaps what is needed is to evaluate genes as members of gene pathways, and use the pathway information to somehow guide the selection of predictive genes. Ideally, one would like to have detailed gene pathway information, which can then be used to select genes with a potential causal link to cancer and metastasis. This has largely not been possible due to limitations on data size (too few samples) and the complexity of gene interactions. Therefore, the problem of finding the pathway information must be tackled in other ways. One way is to assume that genes with correlated expression belong together in one pathway (or are somehow otherwise related to each other, even if they do not interact directly), and to find the sets de novo in the data, using methods such as searching over a space of models representing regulation programs [10] and $k$-means clustering [11]. Similarly, van Vliet et al. [12] used an unsupervised module discovery method to find gene modules, calculated a discrete module activity score, and used the score as feature for a naïve Bayes classifier. They reported that classifiers based on gene sets were slightly better predictors of breast cancer outcome than those based on individual genes. Chuang et al. [13] used a mutual-information scoring approach to analyse known protein-protein interaction (PPI) networks, infer gene pathways, and find subnetworks predictive of breast cancer metastasis.

The other main approach has been to use external pathway information, for example, from the literature. Svensson et al. [14] analysed expression data from ovarian cancers based on gene sets from the Gene Ontology (GO) [15]; to represent the set's expression they used a statistic that is essentially a majority-vote of the over- and under-expressed genes (whether the set is over- or underexpressed on average). In a large study of 12 breast cancer datasets, Kim and Kim [16] reported a classification accuracy of $67.6 \%$ over 6 additional datasets, using 2411 gene sets from GO categories, pathway data, and other sources. They, too, reported low overlap between the top gene sets identified, in terms of their common genes. Lee et al. [17] used the MSigDB C2 gene sets, selected gene sets using the $t$-test on their constituent genes, and used the sets as features for classification in several cancer datasets, including breast cancer. They, did not, however, examine whether features derived from gene sets are any more stable than those based on individual genes, a question which is the focus of our work.

Once a tentative or known gene pathway has been identified, the next issue is how to use the expression levels of its constituent genes in a meaningful way. Some options are to use the mean or median expression [18], the first few principal components [19], and the $z$-statistic [20]. Below we examine several approaches, which we call set statistics.

\section{Our Approach}

In this work we propose using prior knowledge, in the form of pre-specified gene sets from the Molecular Signatures Database (MSigDB) [21] dataset, in order to form new features from the individual genes. Moving away from considering genes in isolation, these features serve as proxies for measuring the activity of the set as a whole. There are many approaches to gene set enrichment [22]; however, it is not clear whether these enrichment measures imply good predictive abilities. In contrast, we compare features derived from gene sets with features based on individual genes, with respect to the following criteria:

- discrimination: ability to predict metastasis within 5 years, both on average and its variance;

- stability of the ranks of individual features within datasets;

- concordance between the weights and ranks of features from different datasets;

- and the underlying biological process pointed to by the features.

\section{Methods}

We explored a range of methods for extracting gene sets. These statistics are described below; we first discuss the data used.

\section{Data}

We used five breast cancer datasets from NCBI GEO [23]: GSE2034 [24], GSE4922 [25], GSE6532 [26,27], GSE7390 [28], and GSE11121 [29]. All five are Affymetrix HG-U133A microarrays (some datasets include other platforms; these platforms were excluded). We removed quality control probesets, probesets with close to zero variance across samples, and probesets with more than $15 \%$ missing expression levels (otherwise, missing values were imputed using the median expression of the nonmissing samples [[30], pp. 48-50]). In total, each microarray had 22,215 remaining probesets. The datasets were independently normalised (see Additional File 1). 


\section{Data Composition}

The data contains both lymph-node-negative and nodepositive breast cancer patients. For GSE7390, GSE11121, and GSE2034, none of the patients received adjuvant treatment. For GSE6532 and GSE4922, some patients received adjuvant therapy; these were removed from the data. The data contains patients with both ER-positive and ER-negative tumours. Patients were classified into two groups, low and high risk, according to the time to distant metastasis, using a cutoff point of 5 years. Patients censored before the cutoff were considered noninformative and were removed from the data, as shown in Table 1.

\section{Gene Sets}

We used five MSigDB http://www.broadinstitute.org/ gsea/msigdb gene set collections: $\mathrm{C} 1$ (positional gene sets), C2 (curated gene sets), C3 (motif gene sets), C4 (computational gene sets), and C5 (GO terms) [21], a total of 5452 gene sets, of which 5414 sets could be mapped to the Affymetrix HG-U133A probesets. Note that the $\mathrm{C} 2$ collection includes sets derived from KEGG [31] and Gene Ontology [15], among others.

\section{Set Statistics}

The purpose of the set statistic is to reduce the set's expression matrix to a single vector, which is then used as a feature for classification. The intention is for the set statistic to be representative of the expression levels of the set, in a useful way. Here we describe the different set statistics used in this work. All of our set statistics are $u n s u$ pervised, in the sense that they do not take into account the metastatic class, unlike methods such as the $t$-test [32], GSEA [21], or GSA [33]; any standard classifier, such as a support-vector machine (SVM), can be built on top of these features.

\section{Mathematical Notation}

Here, $\mathbf{X}$ is the $p \times N$ matrix of gene expression levels, where $p$ denotes the number of genes and $N$ denotes the number of samples. Every gene belongs to one or more gene sets $\mathcal{S}_{j}$, such that $\mathcal{S}_{j} \varepsilon\{1, \ldots, p\}, j=1, \ldots, M$, where $M$ is the number of gene sets. $s_{j}=\left|\mathcal{S}_{j}\right|$ and $s_{\neg j}$ denote the number of genes inside and outside the $j$ th set, respectively.

\section{Set Centroid and Set Median}

The centroid is simply the mean expression level over all genes in the set. The matrix of all centroids is an $M \times N$ matrix with entries

$$
\text { centroid }_{j i}=\frac{1}{\mathcal{S}_{j}} \sum_{\left\{k \in \mathcal{S}_{j}\right\}} x_{k i},
$$

where $x_{k i}$ is the expression level for the $k$ th gene in the $i$ th sample. Similarly, the set median is the median expression level for all genes in the set, for a given sample.

The motivation for the centroid is that it reduces the variance of noise in the feature, since the sample variance of the mean of a random vector $\boldsymbol{x}=\left(x_{1}, \ldots, x_{n}\right)$ is the square of the standard error of the sample mean $\bar{x}$,

$$
\operatorname{Var}(\bar{x})=\left[\frac{\operatorname{sd}(x)}{\sqrt{n}}\right]^{2}=\frac{\operatorname{Var}(x)}{n}<\operatorname{Var}(x),
$$

for $n>1$. The actual decrease in variance depends on the degree of correlation of the variables. Another interpretation is that all the genes in the same set are effectively shrunk towards the set mean, thereby reducing the effect of outlier genes [32] and reducing overfitting. The set median is similar to the centroid, except that it is less sensitive to outliers.

\section{Set Medoid}

The matrix of medoids is defined as the gene in the set $S_{j}$ closest (in Euclidean distance) to the centroid, for each sample $i$

Table 1: Sample sizes and breakdown by class

\begin{tabular}{lcccc}
\hline Dataset & $<$ years & $\begin{array}{c}\text { Good Obs. } \\
\geq \mathbf{5} \text { years }\end{array}$ & Total & Removed Obs. \\
\hline GSE2034 & 82 & 165 & 247 & 8 \\
GSE4922 & 30 & 103 & 133 & 9 \\
GSE6532 & 21 & 91 & 112 & 25 \\
GSE7390 & 36 & 154 & 190 & 8 \\
GSE11121 & 28 & 154 & 182 & 18 \\
\hline
\end{tabular}

Observations (samples) were removed if they were censored before the 5-year cutoff. 


$$
\operatorname{medoid}_{j i}=\underset{k \in \mathcal{S}_{j}}{\arg \min }\left(x_{k i}-\text { centroid }_{j i}\right)^{2},
$$

where $x_{k i}$ is the expression level for the $k$ th gene (out of the $s_{j}$ genes in the set) in the $i$ th sample. In this formulation, the set medoid is not the same as the set median.

\section{Set t-Statistic}

The set centroid does not take into account different means and variances between the genes, nor the fact that a gene may have a high mean but high variance as well (low signal-to-noise ratio). An alternative is to use the one-sample $t$-statistic. The matrix of $t$-statistics is computed by first centering and scaling the expression matrix so that each gene has mean zero and unit variance, and then computing the $t$-statistic for each set in each sample,

$$
t_{j i}=\frac{\operatorname{centroid}_{j i} \sqrt{s_{j}}}{\operatorname{sd}_{j i}},
$$

where centroid ${ }_{j i}$ is the centroid statistic from Eq. 1, and $\mathrm{sd}_{j i}$ is the standard deviation of the genes in set $j$ in the $i$ th sample. Scaling is done to prevent spurious $t$-statistics, due to very small variances, from inflating the importance of "non-interesting" genes. We excluded sets with fewer than 30 genes.

\section{$U$-statistic -value}

The competitive U-statistic for the set, also known as Wilcoxon's rank-sum statistic [35], compares the mean rank of the genes in the set to the mean rank of the genes outside the set, for all samples. The U-statistic is calculated as follows.

1. Create a list of gene expression ranks $L_{i}=\left\{l_{i 1}, l_{i 2}, \ldots, l_{i s_{j}}\right\}$ of the $s_{j}$ genes in the set in the $i$ th sample.

2. Sum the ranks for the set $R_{i j}=\sum_{k=1}^{s_{j}} L_{k}$

3. The U-statistic for set $S_{j}$ in sample $i$ is then $U_{i j}=R_{i j}$ $s_{j}\left(s_{j}+1\right) / 2$.

For large $n$, the $j$ th U-statistic is approximately normally-distributed, with $\mu=s_{j} s_{\neg j} / 2$ and $\sigma^{2}=s_{j} s_{\neg j}\left(s_{j}+s_{\neg j}+1\right) /$ 12. Once the U-statistic is computed, we use its $\log p$ value as the feature for the classifier.

This statistic is slightly unusual since it pits gene sets against other gene sets, that is, its distribution depends on the number of genes sets rather than samples. Goeman and Bühlmann [36] argue that this statistic is inappropriate since it switches the standard relationship between genes and samples in the experimental setup (the sample size becomes the number of genes, not the number of microarrays); however, Barry et al. [37] consider it a useful statistic nonetheless. In any case, we use this statistic only as a feature for a classifier, and not to directly make inferences about the statistical significance of the sets' expression levels.

\section{1st Principal Component of the Set}

Principal Component Analysis (PCA) is performed using the singular value decomposition (SVD) of the gene set's $s_{j} \times N$ expression matrix $\mathbf{X}_{j}$, defined as

$$
\mathbf{X}_{j}^{T}=\mathbf{U}_{j} \mathbf{D}_{j} \mathbf{V}_{j}^{T},
$$

where $\mathbf{U}_{j}$ and $\mathbf{V}_{j}$ are matrices whose columns are the left and right singular vectors, respectively, and $\mathbf{D}_{j}$ is a diagonal matrix with the diagonal being the eigenvalues (also called loadings).

The first eigenvector $\mathbf{v}_{1 j}$ (first column of $\mathbf{V}_{j}$ ) explains the highest amount of variance in $\mathbf{X}_{j}^{T}$. The 1st principal component $(\mathrm{PC})$ of the matrix, $\mathrm{PC} 1_{j}$, is obtained by projecting the data onto that eigenvector

$$
\mathrm{PC}_{j}=\mathbf{X}_{j}^{T} \boldsymbol{v}_{1 j}
$$

where $\boldsymbol{v}_{1 j}$ is an $s_{j} \times 1$ column vector. Hence, PC1 is the best rank-1 approximation of the data. We mean-centred and scaled the matrix $\mathbf{X}^{T}$ before applying PCA.

\section{The Centroid Classifier}

Feature instability, manifested as discordant gene lists, can be caused both by inherent instability and by overfitting the classifier to the data. Therefore, to reduce the risk of overfitting, we use the centroid classifier [[38], pp. 4-6]. The centroid classifier is equivalent to a heavily-regularised support vector machine [39] and to Fisher Linear Discriminant Analysis (LDA) with diagonal covariance and uniform priors $[40,41]$. Therefore, we expect that it is less prone to overfitting than an SVM or similar classifiers. We further stabilise the centroid's estimate by averaging them over random subsamples of the data, see discussion of "Internal and External Validation."

The centroid classifier finds the centroid of each class, that is, the $p$-vector of average gene expression in each class. New observations are classified by comparing their expression with the two centroids, and choosing the closest centroid. Given a $p \times N$ matrix $\mathbf{Z}$, the centroids ( $p$-vectors) of the positive and negative classes are, respectively,

$$
c^{+}=\frac{1}{n^{+}} \sum_{\left\{i \mid y_{i}=1\right\}} z_{i}, c^{-}=\frac{1}{n^{-}} \sum_{\left\{i \mid y_{i}=-1\right\}} z_{i},
$$

where $n^{+}$and $n^{-}$are the number of samples in each class, and $z_{i}$ is the $i$ th expression vector of $p$ features (ith 
column of $\mathbf{Z}$ ). The centroid classifier predicts using the inner-product rule $\hat{\gamma}_{i}=\left\langle z_{i}-c, w\right\rangle$, where $c=\left(c^{+}+c^{-}\right) / 2$ is the point midway between the centroids, and the weight of each feature is

$$
w=c^{+}-c^{-},
$$

where $w$ is the $p$-vector connecting the two centroids.

The sign of $\hat{y}_{i}$ is then the predicted class. For calculating AUC we use $\hat{\gamma}_{i}$ as the prediction, since it produces an AUC estimate with lower variance than using the class prediction sign $\left(\hat{\gamma}_{i}\right)$.

This centroid classifier is similar but not identical to the classifier used by van 't Veer [1]; they assigned each sample to the class that has the highest Pearson correlation of its centroid with the sample. This is equivalent to our version of the centroid classifier when the samples are scaled to unit norm [[42], p. 203]. See Additional File 1 for further discussion.

Despite its simplicity, the centroid classifier performs well in microarray studies [39] where commonly the number of features is much greater than the number of samples $(p \gg N)$. For the centroid classifier, we observed discrimination similar to or better than several other classifiers (support vector machine, nearest shrunken centroids [40], and the van 't Veer [1] classifiers, see Additional File 1 for details). The centroid classifier requires no tuning, making it fast to compute, and making nested cross-validation unnecessary during feature selection (see [43] for examples of where it is required).

\section{Internal and External Validation}

Since we have five datasets, it might be reasonable to combine them. However, we were interested in measuring the concordance between datasets rather than performing a meta-analysis. The inter-dataset analysis emulates the real-world situation where different studies are performed separately, rather than pooled together. Therefore, we distinguish between internal and external validation. In the former, we estimate the classifier's generalisation inside each dataset, using repeated random subsampling; the subsampling is used to form a bagged classifier for each dataset (see below). We then perform external validation, where the bagged classifier from each dataset is used to predict the metastatic class of patients from another dataset. This is a more realistic estimate of the classifier's discriminative ability.
In the internal validation, we used repeated random subsampling to estimate the classifier's internal generalisation error, as measured by AUC (see Additional File 1 for the AUC from internal validation). In this approach, the dataset is randomly split $B$ times into training and testing parts ( $2 / 3$ and $1 / 3$ of the data, respectively). We used $B=25$ splits. Repeated random subsampling with a $2 / 3-1 / 3$ split is similar to the 0.632 bootstrap without replacement [44]. Each split results in one model; the predictions from $B$ models are then combined into one prediction -- bagging [[45], pp. 246-250] -- by averaging over the $B$ predictions $\hat{\gamma}_{1}, \ldots, \hat{y}_{B}$, and using that vector of averages as the final prediction. Bagging also reduces the variance of the predictions, without increasing the bias [45].

\section{Results and Discussion Classification}

We observed that the discrimination from the internal and external validation were similar, showing that the centroid classifier did not significantly over- or under-fit

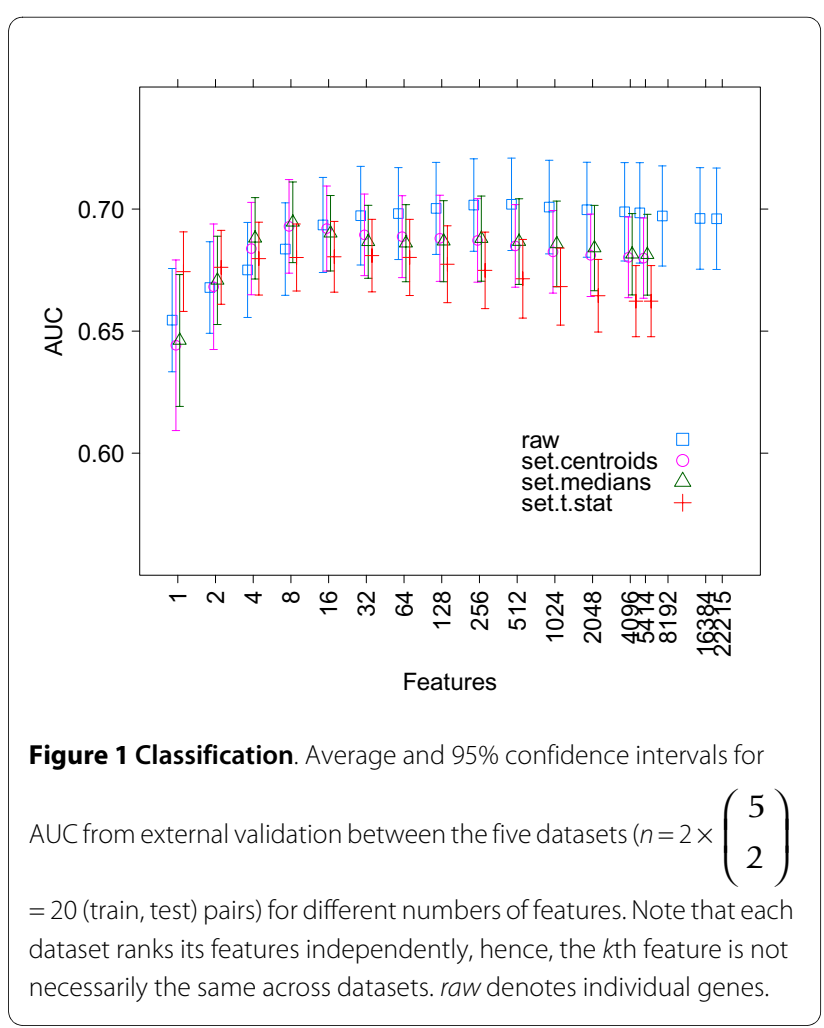


the data. Following are results for the external validation; see Additional File 1 for the internal validation results.

Figure 1 shows AUC for external validation (trained on one dataset and predicted on another, a total of $2\left(\begin{array}{l}5 \\ 2\end{array}\right)=$ 20 predictions), using centroid classifiers trained on different numbers of features. The maximum number of features is 22,215 for genes and for 5414 gene sets. For clarity, we only show the results for classifiers based on expression of individual genes (denoted "raw"), the set centroid, the set median, and set $t$-statistic. (See Additional File 1, Figure 6 for more set statistics and Additional Table 1 for significance tests.) Unlike classifiers such as logistic regression or SVMs, the centroid classifier's weight of one feature does not depend on the others. While it is known that genes are not independently expressed, this strong assumption does not appear to reduce classification accuracy in our datasets. In addition, this assumption makes recursive feature elimination especially simple, since features can be eliminated in reverse order of their rank, where the rank is the absolute value of their weights $w$, and the rank does not need to be recomputed each time. The best AUC of about 0.7 is consistent with previous results based on either lists of individual genes [1,24] or of gene sets [12]. The set centroid, set medoid, set median, and set $t$-statistic showed similar AUC to that of individual genes. The set PC, and set $\mathrm{U}$ statistic showed statistically-significant reductions in AUC compared with individual genes, see Additional Table 1. (Note that each dataset ranks the sets independently, hence the top sets may be different. A consensus list of top sets is provided in Table 2).

While AUC does not seem to improve, on average, by using set statistics rather than individual genes, Figure 2 shows that the variance of the AUC is consistently lower for the set t-statistic than for individual genes. This observation is consistent with the greater stability of gene set features, discussed below.

\section{Stability of Feature Ranks}

We were interested in how the ranks of a single feature vary, since we prefer features that are highly ranked on average and have small variability about that average. If a feature has low average rank and large variability, it may sometimes appear at the top of list simply by chance when the experiment is repeated, indicating that it is not a reliable predictor. Features with high average rank and large variability may appear to be good predictors (on average) but will create unstable gene lists, manifesting as different datasets producing different gene lists of similar predictive ability.

To evaluate the variability of the ranks, we used the percentile bootstrap to sample the observations with replacement, generating a bootstrap distribution for the centroid weights for genes and gene sets in one dataset (GSE4922). Since there are 22,215 genes and only 5414 gene sets, a reduced gene list was derived by training a centroid classifier on the GSE11121 dataset and selecting the 5414 genes with the highest absolute centroid weights; the list was fixed across bootstrap replications.

In many cases we are interested in a small signature comprised of the most useful or predictive features. Therefore, we selected the top 15 genes and gene sets based on their mean rank. Figure 3 shows the mean, 2.5\%, and $97.5 \%$ percentiles from 5000 bootstrap replications, for these top features (shown from highest to lowest) using the set centroid statistic (see Additional Figure 8 for the results for other set statistics). It is clear that the top gene sets have lower variation than the top genes. In light of these results, it is not surprising that lists of prognostic genes show little overlap, as even the best ranked genes vary considerably within the same dataset, let alone between them; gene set features are more stable.

\section{Concordance of Datasets}

We were interested in how the different datasets agreed on the importance of the features (genes or gene sets). We used two approaches: rank-correlation of the centroid classifier's weights, and concordance of the gene lists. For this section, the classifier was not bagged -- we trained a single centroid classifier on each dataset. We note that the datasets are independently normalised -- we are interested in the agreement between datasets despite different normalisation schemes.

We measured concordance using Spearman rank-correlation between the classifier weights estimated from each dataset, a total of $\left(\begin{array}{l}5 \\ 2\end{array}\right)=10$ comparisons. Figure 4 shows the Spearman rank-correlations for each set statistic. It is evident that the rank-correlations for the weights of the set centroids, set median, set medoid, and the set $t$ statistic are higher than for the individual genes. This 
Table 2: Top gene sets by average rank

\begin{tabular}{|c|c|c|c|c|c|}
\hline$\#$ & Set & Cat. & Sign & $\begin{array}{l}\text { MSigDB } \\
\text { Description }\end{array}$ & Enriched GO BP Terms (adj. $p$-value) \\
\hline 1 & GNF2_MKI67 & $\mathrm{C} 4$ & -1 & Neighborhood of MKI67 & $\begin{array}{l}\text { "phosphoinositide-mediated signaling": } 1.95 \times 10^{-10} \text {, "spindle organization": } \\
5.86 \times 10^{-6} \text {, "establishment of mitotic spindle localization": } 1.10 \times 10^{-5}, \\
\text { "kinetochore assembly": } 5.48 \times 10^{-5} \text {, "mitotic chromosome condensation": } 1.37 \\
\times 10^{-4} \text {, "protein complex localization": } 2.55 \times 10^{-3} \text {, "regulation of striated } \\
\text { muscle development": } 2.55 \times 10^{-3} \text {, "metaphase plate congression": } 2.55 \times 10^{-3}\end{array}$ \\
\hline 2 & GNF2_CCNA2 & $\mathrm{C} 4$ & -1 & Neighborhood of CCNA2 & $\begin{array}{l}\text { "phosphoinositide-mediated signaling": } 4.05 \times 10^{-16} \text {, "DNA replication": } 1.04 \times \\
10^{-9} \text {, "mitotic chromosome condensation": } 1.32 \times 10^{-8} \text {, "regulation of striated } \\
\text { muscle development": } 3.76 \times 10^{-3} \text {, "metaphase plate congression": } 3.76 \times 10^{-3}\end{array}$ \\
\hline 3 & GNF2_TTK & $\mathrm{C} 4$ & -1 & Neighborhood of TTK & $\begin{array}{l}\text { "phosphoinositide-mediated signaling": }<2.22 \times 10^{-16} \text {, "mitotic chromosome } \\
\text { condensation": } 4.35 \times 10^{-14} \text {, "DNA replication": } 1.01 \times 10^{-12} \text {, "spindle } \\
\text { organization": } 1.37 \times 10^{-9}, \text { "establishment of mitotic spindle localization": } 9.59 \\
\times 10^{-5} \text {, "kinetochore assembly": } 4.76 \times 10^{-4} \text {, "DNA repair": } 5.78 \times 10^{-3}, \text { "mitosis": } \\
9.44 \times 10^{-3}\end{array}$ \\
\hline 4 & GNF2_HMMR & $\mathrm{C} 4$ & -1 & Neighborhood of HMMR & $\begin{array}{l}\text { "phosphoinositide-mediated signaling": }<2: 22 \times 10^{-16} \text {, "mitotic cell cycle } \\
\text { spindle assembly checkpoint": } 1.26 \times 10^{-11}, \text { "spindle organization": } 4.89 \times 10^{-10} \text {, } \\
\text { "mitotic chromosome condensation": } 8.46 \times 10^{-8}, \text { "cell proliferation": } 6.22 \times 10^{-} \\
\text {6,"DNA replication": } 1.09 \times 10^{-5}, \text {,establishment of mitotic spindle localization": } \\
5.33 \times 10^{-5} \text {, "kinetochore assembly": } 2.65 \times 10^{-4} \text {, "protein complex localization": } \\
8.29 \times 10^{-3} \text {, "regulation of striated muscle development": } 8.29 \times 10^{-3} \text {, } \\
\text { "metaphase plate congression": } 8.29 \times 10^{-3}\end{array}$ \\
\hline 5 & GNF2_CDC20 & C4 & -1 & Neighborhood of CDC20 & $\begin{array}{l}\text { "phosphoinositide-mediated signaling": }<2.22 \text { 10-16, "spindle organization": } \\
2.20 \times 10^{-12} \text {, "mitotic cell cycle spindle assembly checkpoint": } 4.07 \times 10^{-11}, \\
\text { "mitotic chromosome condensation": } 1.52 \times 10^{-9} \text {, "cell proliferation": } 8.96 \times 10^{-} \\
9 \text {, "mitosis": } 1.83 \times 10^{-8} \text {, "establishment of mitotic spindle localization": } 8.95 \times \\
10^{-5} \text {, "kinetochore assembly": } 4.45 \times 10^{-4} \text {, "DNA replication": } 7.83 \times 10^{-3}\end{array}$ \\
\hline 6 & GNF2_SMC2L1 & $\mathrm{C} 4$ & -1 & $\begin{array}{l}\text { Neighborhood of } \\
\text { SMC2L1 }\end{array}$ & $\begin{array}{l}\text { "mitotic cell cycle spindle assembly checkpoint": } 5.15 \times 10^{-13} \text {, "mitotic } \\
\text { chromosome condensation": } 7.16 \times 10^{-9}, \text { "phosphoinositide-mediated } \\
\text { signaling": } 2.14 \times 10^{-6} \text {, "establishment of mitotic spindle localization": } 1.31 \times \\
10^{-5} \text {, "kinetochore assembly": } 6.51 \times 10^{-5} \text {, "protein complex localization": } 2.90 \\
\times 10^{-3} \text {, "DNA strand elongation during DNA replication": } 2.90 \times 10^{-3} \text {, } \\
\text { "regulation of striated muscle development": } 2.90 \times 10^{-3}, \text { "metaphase plate } \\
\text { congression": } 2.90 \times 10^{-3} \text {, "cell proliferation": } 2.94 \times 10^{-3} \text {, "nucleotide-excision } \\
\text { repair, DNA gap filling": } 3.56 \times 10^{-3}\end{array}$ \\
\hline 7 & GNF2_H2AFX & $\mathrm{C} 4$ & -1 & Neighborhood of H2AFX & $\begin{array}{l}\text { "cell proliferation": } 9.28 \times 10^{-10} \text {, "phosphoinositide-mediated signaling": } 5.54 \times \\
10^{-7}, \text { "mitosis": } 8.48 \times 10^{-5}, \text { "mitotic cell cycle spindle assembly checkpoint": } \\
1.33 \times 10^{-4} \text {, "protein complex localization": } 1.63 \times 10^{-3}\end{array}$ \\
\hline 8 & GNF2_ESPL1 & $\mathrm{C} 4$ & -1 & Neighborhood of ESPL1 & $\begin{array}{l}\text { "phosphoinositide-mediated signaling": } 5.38 \times 10^{-11} \text {, "kinetochore assembly": } \\
3.12 \times 10^{-5}, \text { "mitotic chromosome condensation": } 6.75 \times 10^{-5} \text {, "spindle } \\
\text { organization": } 7.76 \times 10^{-4} \text {, "protein complex localization": } 1.67 \times 10^{-3} \text {, } \\
\text { "regulation of striated muscle development": } 1.67 \times 10^{-3}, \text { "metaphase plate } \\
\text { congression": } 1.67 \times 10^{-3}\end{array}$ \\
\hline 9 & GNF2_RRM2 & $\mathrm{C} 4$ & -1 & Neighborhood of RRM2 & $\begin{array}{l}\text { "phosphoinositide-mediated signaling": } 4.52 \times 10^{-15} \text {, "mitotic cell cycle spindle } \\
\text { assembly checkpoint": } 1.17 \times 10^{-9} \text {, "spindle organization": } 1.20 \times 10^{-7} \text {, "DNA } \\
\text { replication": } 5.42 \times 10^{-6}, \text { "cell proliferation": } 1.97 \times 10^{-5} \text {, "establishment of } \\
\text { mitotic spindle localization": } 4.09 \times 10^{-5} \text {, "kinetochore assembly": } 2.03 \times 10^{-4} \text {, } \\
\text { "protein complex localization": } 6.80 \times 10^{-3} \text {, "regulation of striated muscle } \\
\text { development": } 6.80 \times 10^{-3} \text {, "metaphase plate congression": } 6.80 \times 10^{-3}\end{array}$ \\
\hline 10 & GNF2_PCNA & $\mathrm{C} 4$ & -1 & Neighborhood of PCNA & $\begin{array}{l}\text { "phosphoinositide-mediated signaling": }<2.22 \times 10^{-16} \text {, "DNA replication": } 1.47 \\
\times 10^{-15} \text {, "mitotic chromosome condensation": } 2.36 \times 10^{-7} \text {, "spindle } \\
\text { organization": } 4.33 \times 10^{-7} \text {, "establishment of mitotic spindle localization": } 9.59 \\
\times 10^{-5} \text {, "cell proliferation": } 4.18 \times 10^{-4} \text {, "DNA repair": } 4.33 \times 10^{-4} \text {, "kinetochore } \\
\text { assembly": } 4.76 \times 10^{-4} \text {, "mitosis": } 9.44 \times 10^{-3}\end{array}$ \\
\hline
\end{tabular}

Top 10 gene sets by average rank over the five datasets, using the set centroid statistic. GO enrichment $p$-values are from a Bonferroni-adjusted one-sided Fisher's exact test (30,330 tests). Sign $=-1$ if expression is negatively associated with long-term survival, and vice versa. The background list for the test includes all Affymetrix HG-U133A probesets that could be mapped to GO BP terms, excluding IEA annotations. 


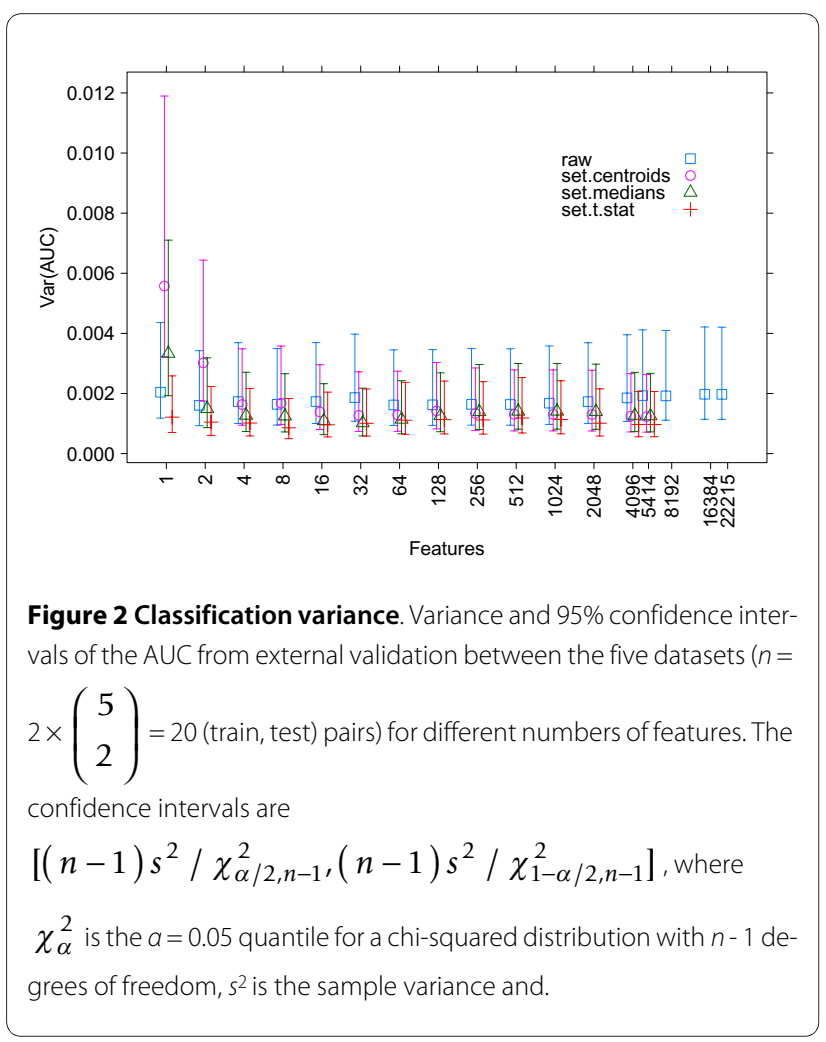

indicates that classifiers built from features based on gene sets are more stable than those built using individual genes, and are less likely to overfit.

To measure how the ranked lists produced by each dataset agreed on the top-ranked genes, we used the following approach. The features for each dataset were ranked by the absolute value of their $w$ weight. Then, for each number $f$ of features, $f=1, \ldots, p$, we chose each dataset's top $f$-ranked features. Next, we counted how many of these $f$ features occurred at least $k$ of the five datasets. Results for $k=5$ are shown in Figure 5 . Lists based on individual genes show little overlap for cutoffs up to about 130 -- in other words, there are no genes that occur in all five datasets up to that cutoff. In comparison, the set statistics, especially the set medians and the set centroids, produce lists with higher overlap, even at cutoffs below 10. This result further supports the conclusion that lists of individual genes are highly unstable, and that the little overlap between reported prognostic signatures is to be expected.

\section{MSigDB Sets}

Table 2 shows the top 10 gene sets by rank, where the rank was averaged over the feature ranks from the five datasets, using the set centroid statistic. Also shown is enrichment for BO Biological Process (BP) terms from a Bonferroni-adjusted Fisher's exact test, for the genes

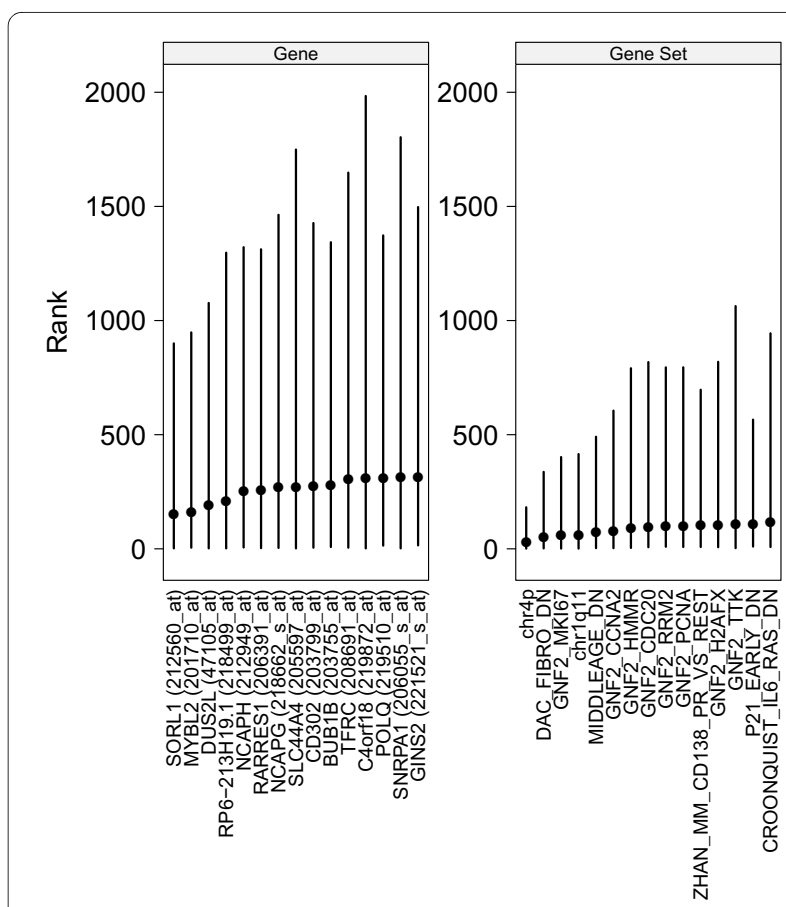

Figure 3 Bootstrap. Mean and 2.5\%/97.5\% of the ranks of genes and gene sets (set centroid statistic), over 5000 bootstrap replications of the GSE4922 dataset. The features have been sorted by their mean rank.

belonging to these sets. The top sets are enriched for GO BP terms related to the cell cycle and cell division processes, and for the PI3K pathway which interacts with the Ras oncogene [46], confirming the cell cycle process as one of the major biological mechanisms associated with breast cancer metastasis [47,48]. The top set,

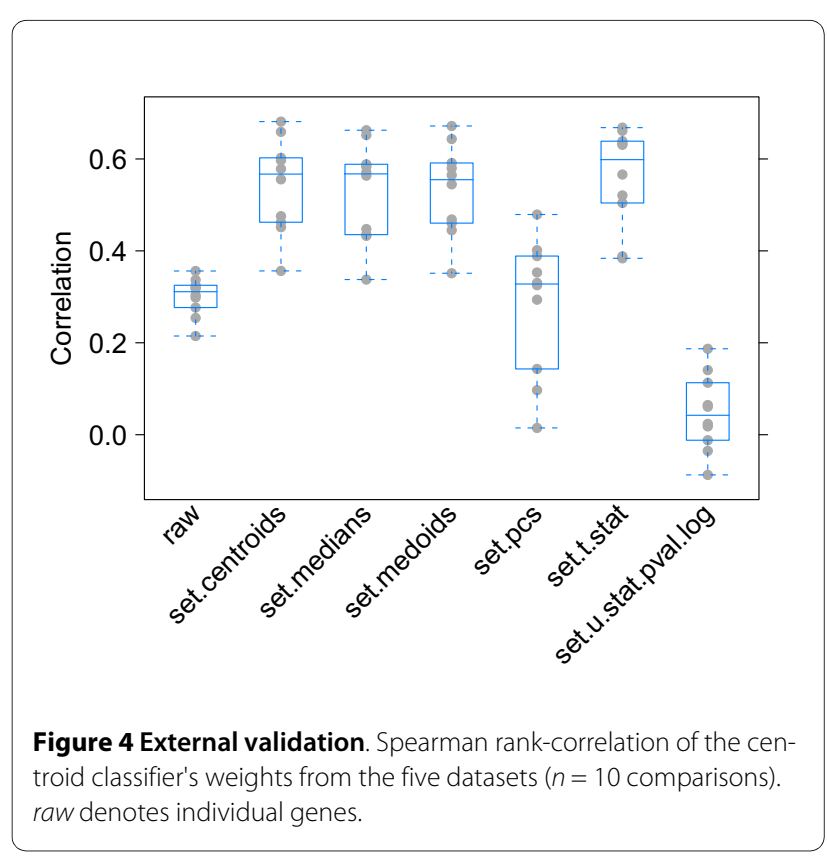




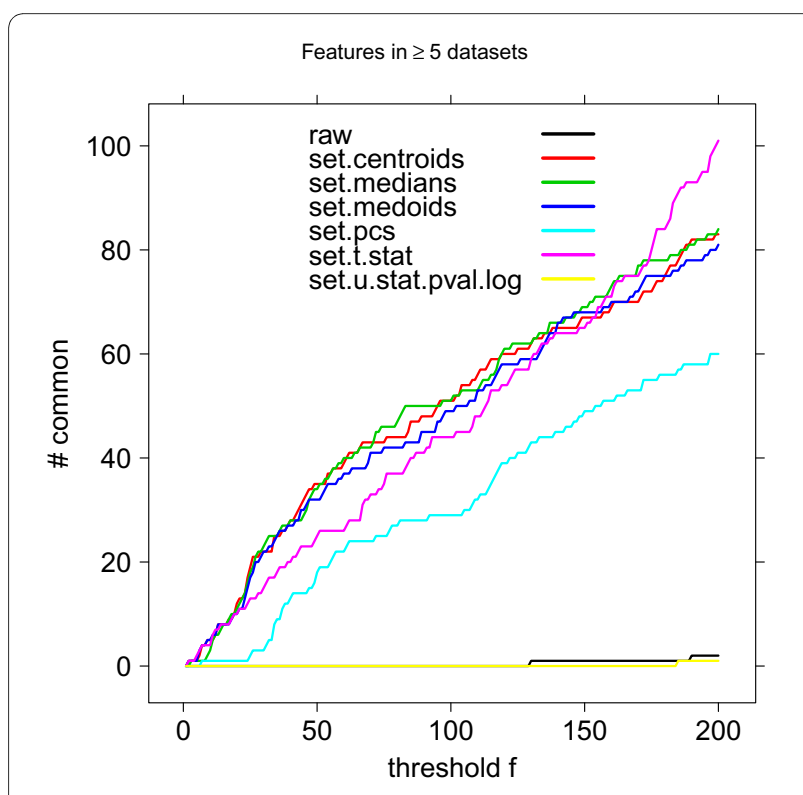

Figure 5 List concordance. Concordance of feature lists (genes or gene sets) for different cutoffs $f=1, \ldots, 200$, counting the number of features occurring in all of the five datasets' lists, ranked higher than $f$. raw denotes individual genes.

GNF2_MKI67, is related to Ki-67, a known marker of cancer proliferation [49].

The potential advantage of gene sets signatures over individual gene signatures depends on the degree of these genes' coexpression. A critical aspect of this performance, therefore, is the source for the grouping of genes into sets. The MSigDB is composed of five set classes depending on the annotation used to define the sets. Whereas categories $\mathrm{C} 1$ and $\mathrm{C} 3$ are derived from the chromosomal location and sequence of regulatory elements, respectively, categories $\mathrm{C} 2$ and $\mathrm{C} 4$ both originate from expression profiles; $\mathrm{C} 5$ is based on $\mathrm{GO}$ categories. In addition, the datasets these categories are based on vary with respect to sample size; whereas $\mathrm{C} 4$ was is based on hypothesis-free examination of co-expression across almost two thousand expression profiles, $\mathrm{C} 2$ is mainly based on publications of expression profiles, rarely using more than dozens of samples.

To see whether different MSigDB categories were more useful for predicting metastasis, we combined four datasets (GSE2034, GSE4922, GSE6532, and GSE7390) into a single training set. A separate centroid classifier was trained on each gene set, using the set-centroid statistic, and the gene sets were then ranked by their centroid classifier weights (negative to positive). We then tested the classifiers on the fifth dataset, GSE11121. Finally, we used the two-sample Kolmogorov-Smirnov statistic to compare the ranks from the different categories (see Additional File 1 for details and results for other set statistics).

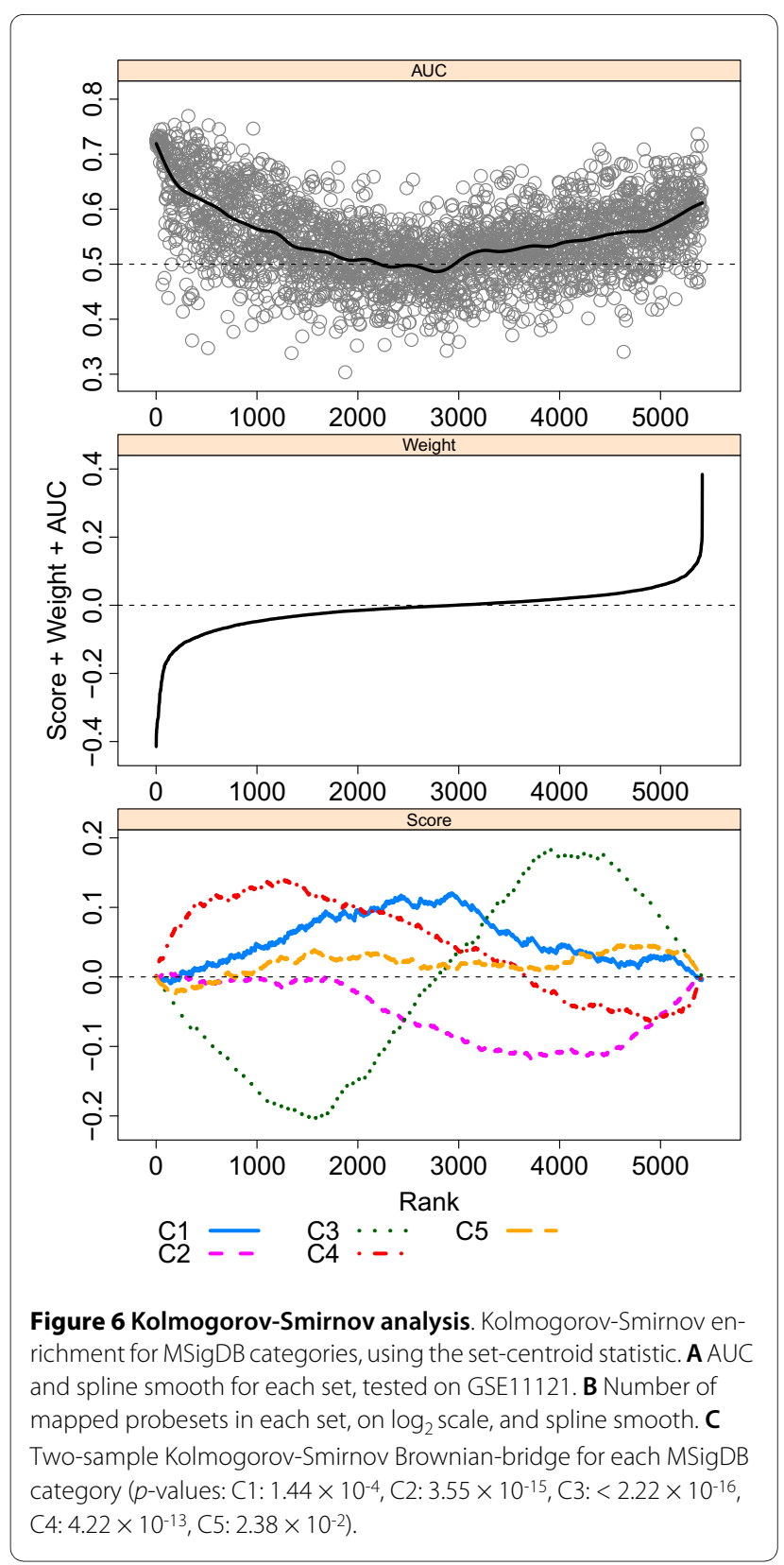

Figure 6 shows the cumulative-sum statistic, from which the Kolmogorov-Smirnov statistic is computed, for the ranked gene sets. In order to link that rank with performance in sample classification, we plotted the classifier's AUC value for each of these sets along the rank. The results show that the $\mathrm{C} 4$ sets tend have extreme centroid weights, especially towards the negative side, than the other categories. In contrast, $\mathrm{C} 2$ sets show a concentration towards the positive weights, albeit much smaller. Category C3 tends to be concentrated in the middle ranks, and category $\mathrm{C} 1$ tends to be concentrated in the negative to middle ranks. Finally, category $\mathrm{C} 5$ is distributed more uniformly across the ranks; this may be since GO sets do not take the direction of expression change 
into account, potentially leading to sets composed of genes with a mixture of positive and negative correlations.

One problem with the set-centroid statistic is that for small sets, there is a higher probability of observing an extreme statistic by chance, since the variance of the sample mean decreases with set size. This implies that spurious set centroids (high absolute value) would be more common in smaller sets, leading to a bias towards smaller sets when ranking the sets. However, there does not seem to be a monotonic relationship between log-size and rank (see Additional File 1, Figure 10). Additionally, there is reasonable concordance between the sets as independently ranked by the five datasets. We conclude that while spurious effects due to the set-size cannot be ruled out, they does not seem to be a major factor in the set's rank. In any case, as an alternative to the set centroid, the set $t$-statistic can be used.

\section{Prognostic Gene Sets in Breast Cancer Molecular Subtypes}

Breast cancer is a heterogeneous disease, with gene expression segregating the cases into different biological and clinically relevant subgroups, perhaps implying differing biological mechanisms for tumour growth and progression and suggesting separate cells of origin. It is reasonable to ask the question whether there is different aetiology related to breast cancer progression. The common molecular classification describes five classes -basal-like, luminal A and B, normal breast tissue-like, and ERBB2+ [50]. Our results above show a strong cell-cycle signature as highly prognostic, supporting existing data [51]. The association of cell-cycle genes to increased risk of metastasis has been mainly attributed to the breast cancer cases that are ER+ [26,52], which comprise the majority of the breast cancer population. Therefore, we explored whether different signatures could be found by removing such cases and training the classifiers on the remaining samples.

The triple-negative class, also called basal-like, is a group of breast cancer that are ER (also known as ESR1), PR (progesterone receptor), and HER2 (also known as ERBB2) [53]. We sought to reproduce the same biological results as previous reports which have identified subtypespecific signatures that predict metastasis $[51,54]$. We followed the procedure described by Desmedt et al. [51],

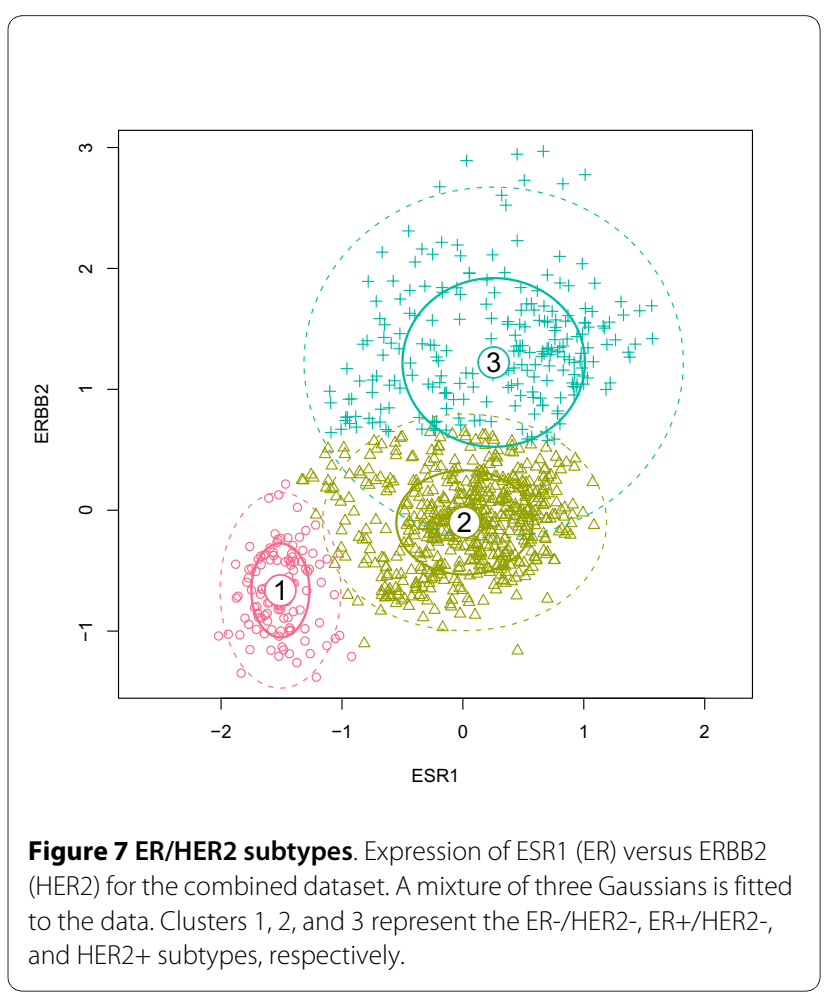

and assessed their list of gene modules which are intended to represent different biological functions such as tumor invasion, immune response, angiogenesis, apoptosis, proliferation, and ER and HER2 signaling (see Additional File 1 for details). We clustered the samples based on their ER and HER2 module scores into three subgroups, ER-/HER2-, ER+/HER2-, and HER2+, shown in Figure 7 and Table 3.

We reran our analysis, consisting of training the centroid classifier on the MSigDB set statistics, on each subgroup. Table 4 shows the top gene sets for each subgroup for the set centroid statistic (see Additional File 1 for others statistics). The set centroid, set medoid, and set median show enrichment for genes from the AURKA module in the ER+/HER2- as expected, and to a lesser extent an immune response signature (STAT1 module) in ER-/HER2-, manifesting as IFN- $\gamma$-related sets in Table 4. Only in the ER-/HER- subgroup did these set statistics result in substantially different top gene sets. We also plotted the Kolmogorov-Smirnov statistics for how enriched were all $5414 \mathrm{MSigDB}$ sets in genes from each of

Table 3: Breakdown of samples for each cancer subtype

\begin{tabular}{llccc}
\hline & Class & $<\mathbf{5}$ years & $\mathbf{2 5}$ years & Total \\
\hline 1 & & & 80 & 115 \\
2 & ER-/HER2- & 35 & 423 & 530 \\
3 & ER+/HER2- & 107 & 164 & 219 \\
\hline
\end{tabular}


Table 4: Top gene sets for each ER/HER2 subtype

\begin{tabular}{|c|c|c|c|c|c|}
\hline Class & $\#$ & MSigDB Set & Cat. & Description & Sign \\
\hline \multirow[t]{10}{*}{ ER-/HER2- } & 1 & chr7q12 & $\mathrm{Cl}$ & Genes in cytogenetic band chr7q12 & 1 \\
\hline & 2 & COLLER_MYC_DN & $\mathrm{C} 2$ & $\begin{array}{l}\text { Genes down-regulated by MYC in } 293 \text { T (transformed } \\
\text { fetal renal cell). }\end{array}$ & -1 \\
\hline & 3 & IFNGPATHWAY & $\mathrm{C} 2$ & IFN gamma signaling pathway & 1 \\
\hline & 4 & GRANDVAUX_IFN_NOT_IRF3_UP & $\mathrm{C} 2$ & $\begin{array}{l}\text { Genes up-regulated by interferon-alpha, beta but not } \\
\text { by IRF3 in Jurkat (T cell) }\end{array}$ & 1 \\
\hline & 5 & GNF2_ST13 & $\mathrm{C} 4$ & Neighborhood of ST13 & -1 \\
\hline & 6 & GNF2_CD48 & $\mathrm{C} 4$ & Neighborhood of CD48 & 1 \\
\hline & 7 & GNF2_GLTSCR2 & $\mathrm{C} 4$ & Neighborhood of GLTSCR2 & -1 \\
\hline & 8 & MENSE_HYPOXIA_DN & $\mathrm{C} 2$ & $\begin{array}{l}\text { List of Hypoxia-suppressed genes found in both } \\
\text { Astrocytes and HeLa Cells }\end{array}$ & -1 \\
\hline & 9 & HSA03010_RIBOSOME & $\mathrm{C} 2$ & Genes involved in ribosome & -1 \\
\hline & 10 & GCM_TPT1 & $\mathrm{C} 4$ & Neighborhood of TPT1 & -1 \\
\hline \multirow{10}{*}{$\begin{array}{l}\text { ER+/ } \\
\text { HER2- }\end{array}$} & 1 & GNF2_MKI67 & $\mathrm{C} 4$ & Neighborhood of MKI67 & -1 \\
\hline & 2 & GNF2_TTK & $\mathrm{C} 4$ & Neighborhood of TTK & -1 \\
\hline & 3 & GNF2_HMMR & $\mathrm{C} 4$ & Neighborhood of HMMR & -1 \\
\hline & 4 & GNF2_CCNA2 & $\mathrm{C} 4$ & Neighborhood of CCNA2 & -1 \\
\hline & 5 & GNF2_SMC2L1 & $\mathrm{C} 4$ & Neighborhood of SMC2L1 & -1 \\
\hline & 6 & GNF2_ESPL1 & C4 & Neighborhood of ESPL1 & -1 \\
\hline & 7 & GNF2_CDC20 & $\mathrm{C} 4$ & Neighborhood of CDC20 & -1 \\
\hline & 8 & GNF2_H2AFX & C4 & Neighborhood of H2AFX & -1 \\
\hline & 9 & GNF2_RRM2 & C4 & Neighborhood of RRM2 & -1 \\
\hline & 10 & ZHAN_MM_CD138_PR_VS_REST & $\mathrm{C} 2$ & $\begin{array}{l}50 \text { top ranked SAM-defined over-expressed genes in } \\
\text { each subgroup_PR }\end{array}$ & -1 \\
\hline \multirow[t]{10}{*}{ HER2+ } & 1 & chr4p & $\mathrm{Cl}$ & Genes in cytogenetic band chr4p & -1 \\
\hline & 2 & chrlqll & $\mathrm{Cl}$ & Genes in cytogenetic band chrlqll & 1 \\
\hline & 3 & DAC_FIBRO_DN & $\mathrm{C} 2$ & $\begin{array}{l}\text { Downregulated by DAC treatment in LD419 fibroblast } \\
\text { cells }\end{array}$ & -1 \\
\hline & 4 & GNF2_MKI67 & $\mathrm{C} 4$ & Neighborhood of MKI67 & -1 \\
\hline & 5 & GNF2_CCNA2 & $\mathrm{C} 4$ & Neighborhood of CCNA2 & -1 \\
\hline & 6 & GNF2_TTK & C4 & Neighborhood of TTK & -1 \\
\hline & 7 & GNF2_H2AFX & $\mathrm{C} 4$ & Neighborhood of H2AFX & -1 \\
\hline & 8 & GNF2_HMMR & $\mathrm{C} 4$ & Neighborhood of HMMR & -1 \\
\hline & 9 & CROONQUIST_L6_RAS_DN & $\mathrm{C} 2$ & $\begin{array}{l}\text { Genes dowmregulated in multiple myeloma cells } \\
\text { exposed to the pro-proliferative cytokine IL- } 6 \text { versus } \\
\text { those with } \mathrm{N} \text {-ras-activating mutations. }\end{array}$ & -1 \\
\hline & 10 & CROONQUIST_L6_STARVE_UP & $\mathrm{C} 2$ & $\begin{array}{l}\text { Genes upregulated in multiple myeloma cells exposed } \\
\text { to the pro-proliferative cytokine IL- } 6 \text { versus those that } \\
\text { were IL-6-starved. }\end{array}$ & -1 \\
\hline
\end{tabular}

Top 10 MSigDB sets for ER/HER2 molecular subtypes, chosen by the centroid classifier using the set centroid statistic. Sign = -1 if expression is negatively associated with long-term survival, and vice versa. 
the modules from [51] (Additional File 1, Figure 11), showing that in contrast to the other set statistics, the set $\mathrm{PC}$, set $\mathrm{t}$-statistic and to some extent the set $\mathrm{U}$ statistic, exhibit more pronounced enrichment of Desmedt's module genes at the top and bottom of the sorted set list, indicating that the sets with large weight, either positive or negative, contain more of the genes defined in Desmedt's modules, and suggesting the same underlying biology as the modules.

Do the Gene Sets Point to the Same Biology As the Genes? In this section we investigate whether the top gene sets reflect the same underlying biology as the top genes. In the combined data, we trained three types of classifier:

Table 5: Overlap between top genes and gene sets for different classifiers

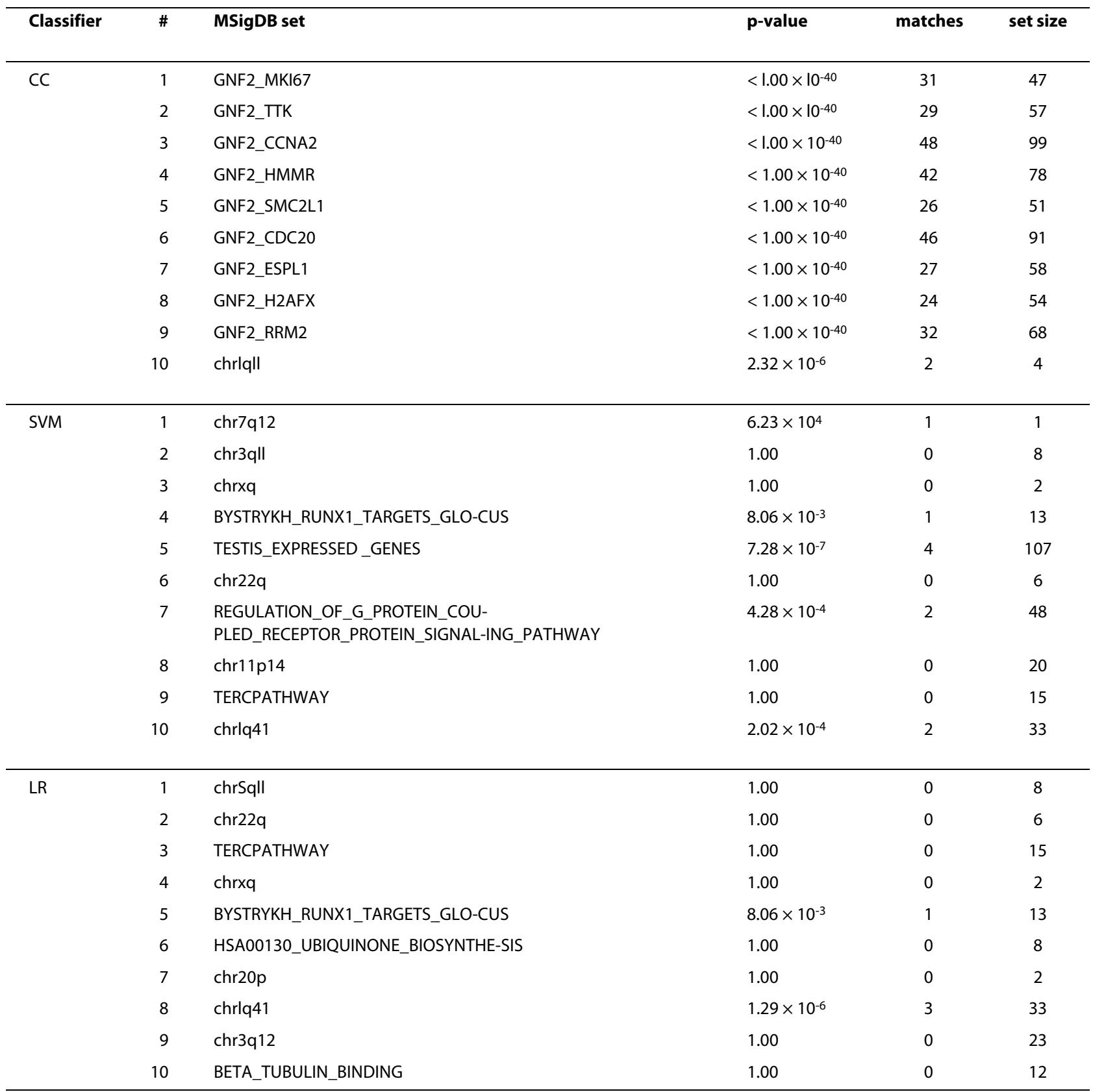

Top 10 sets using the set centroid statistic using different classifiers, and the $p$-value for the number of top genes belonging to each of them (Fisher's exact test, one sided). CC is centroid classifier, LR is logistic regression. 
$<_{2}$-penalised logistic regression ( $\mathrm{R}$ package penalized[55]), SVM with linear kernel (R package kern$l a b[56])$, and the centroid classifier. Each classifier was trained on the genes and the gene set statistics (set centroids, see Additional File 1 for others), for a total of six models.

For each model, we ranked the features by the absolute value of their weights. We then selected the top 512 genes, which is a high enough number of genes producing a high AUC after which AUC does not increase much, and is a much higher number of genes than many published metastatic signatures. Other cutoffs $(256,1024$, 2048) exhibited similar results (not shown). For each of the top ranked sets, we then checked how many of the top ranked genes belonged to that set, using the same classifier (i.e., centroid genes to centroid sets, logistic regression genes to logistic regression sets, SVM genes to SVM sets). The number of genes belonging to each set was quantified using a one-sided Fisher exact test.

As shown in Table 5, there is significant overlap between the top sets and top genes found by the centroid classifier. In comparison, both logistic regression and the SVM show very little overlap. In other words, the top sets ranked by the centroid classifier, using the set centroid statistic, are over-represented in the top genes selected by the centroid classifier, indicating the same underlying biological processes associated with metastasis.

\section{Conclusions}

We have shown that classifiers based on sets, rather than individual genes, have equivalent predictive power but are more stable, and as a result may facilitate increased understanding of the biological mechanism relating to breast cancer prognosis. The likely explanation is that the expression of any given gene is a function of both its contextual regulation -- regulation under varying conditions both observed and unobserved (such as noisy transcription) -- as well as inherent variability due to germ-line variations and differences in host-tumour response between individuals [57]. The former variability can be used for prognostic purposes. However, the latter reduces the prognostic accuracy since patient-level variability is typically not considered when building prognostic models.

Furthermore, the $\mathrm{C} 4$ sets tended to produce better classifiers than the other MSigDB categories. This difference may be due to the fact that $\mathrm{C} 4$ sets are based on datasets with a large number of samples; however, other factors cannot be ruled out. Our results suggest that there is prognostic value in large-scale systematic efforts to compile sets of coexpressed genes $[10,58]$.
Importantly, our results are in agreement with current understanding of the drivers of metastasis in breast cancer -- proliferation for ER+/HER2-, immune response for ER-/HER2-, and tumour invasion and immune response for HER2 + [51] -- suggesting our approach could be a useful one. Apart from patient prognosis, there is also potential for understanding the biological mechanisms responsible for response and resistance to anti-cancer therapies.

We have used simple set statistics to represent gene set activity. These statistics are computationally tractable and depend on predefined set memberships. Some set statistics are not always sensible; for example, the average expression of a gene set of may not be meaningful when the genes are negatively correlated or uncorrelated; different statistics may be optimal for different gene sets. Moreover, these statistics ignore the structure and dynamics of the genetic networks, which could be important in deciphering causal relationships between genes and phenotypes. However, reliable information about the detailed structure of human genetic networks is currently limited.

\section{Availability and requirements}

Project name: Gene Set Statistics

Project homepage: http://www.csse.unimelb.edu.au/ حgabraham/GSS

Operating system(s): Unix-like

Programming languages: $R$

License: GPL v3

Any restrictions for use by non-academics: none

\section{Additional material}

Additional file 1 supplementary. Further details on data preprocessing methodology, and results including internal validation and comparisons of the centroid classifier with other classifiers.

\section{Abbreviations}

PCA: Principal Component Analysis; SVD: Singular Value Decomposition; SVM: Support Vector Machine; MSigDB: Molecular Signatures Database; AUC: Area Under Receiver Operating Characteristic Curve.

\section{Authors' contributions}

GA developed and implemented the methodology, and analysed the data. AK, $\mathrm{IH}$, and $\mathrm{JZ}$ participated in developing the methodology and the analysis of the data. SL participated in the analysis of the data. All authors contributed to the preparation of the manuscript, and read and approved the final manuscript.

\section{Acknowledgements}

This work was supported by the Australian Research Council, and by the NICTA Victorian Research Laboratory. NICTA is funded by the Australian Government as represented by the Department of Broadband, Communications and the Digital Economy and the Australian Research Council through the ICT Center of Excellence program.

$\mathrm{SL}$ is supported by the National Health and Medical Research Council of Australia (NHMRC) and the European Society of Medical Oncology. 
We thank Justin Bedo for providing R code for SVMs, Raj Gaire for critical reading of the manuscript, and William Webber for discussions of ranking methods.

\section{Author Details}

'Department of Computer Science and Software Engineering, The University of Melbourne, Parkville 3010, VIC, Australia, 2NICTA Victoria Laboratory, The University of Melbourne, Parkville 3010, VIC, Australia, ${ }^{3}$ Department of Translational Research and Functional Genomics Unit, Jules Bordet Institute, Brussels, Belgium, ${ }^{4}$ Department of Medical Oncology, Peter MacCallum Cancer Centre, East Melbourne, VIC 3002, Australia, 5 Metastasis Research Laboratory, Peter MacCallum Cancer Centre, East Melbourne, VIC 3002, Australia, ${ }^{6}$ The Blood and DNA Profiling Facility, Baker IDI Institute, Prahran, VIC 3004, Australia and 7 Department of Biochemistry, School of Medicine, University of Melbourne, VIC 3010, Australia

Received: 8 February 2010 Accepted: 25 May 2010

Published: 25 May 2010

\section{References}

1. van't Veer LJ, Dai H, Vijver MJ van de, He YD, Hart AAM, Mao M, Peterse HL, Kooy K van der, Marton MJ, Witteveen AT, Schreiber GJ, Kerkhoven RM, Roberts C, Linsley PS, Bernards R, Friend SH: Gene expression profiling predicted clinical outcome of breast cancer. Nature 2002, 415:530-536.

2. Vijver MJ van de, He YD, van 't Veer $\sqcup$, Dai H, Hart AAM, Voskuil DW, Schreiber GJ, Peterse JL, Roberts C, Marton MJ, Parrish M, Atsma D, Witteveen A, Glas A, Delahaye L, Velde T van der, Bartelink H, Rodenhuis S, Rutgers ET, Friend SH, Bernards R: A gene-expression signature as a predictor of survival in breast cancer. New Eng/ J Med 2002, 347:1999-2009

3. Ein-Dor L, Kela I, Getz G, Givol D, Domany E: Outcome signature genes in breast cancer: is there a unique set? Bioinformatics 2005, 21:171-178.

4. Michiels S, Koscielny S, Hill C: Prediction of cancer outcome with microarrays: a multiple random validation study. The Lancet 2005, 365:488-492.

5. Fan $C$, Oh DS, Wessels $L$, Weigelt $B$, Nuyten DSA, Nobel AB, van't Veer $L$, Perou CM: Concordance among Gene-Expression-Based Predictors for Breast Cancer. N Engl J Med 2006, 355:560-569.

6. Reyal F, van Vliet MH, Armstrong NJ, Horlings HM, de Visser KE, Kok M, Teschendorff AE, Mook S, van 't Veer L, Caldas C, Salmon RJ, Vijver MJ van de, Wessels LFA: A comprehensive analysis of prognostic signatures reveals the high predictive capacity of the Proliferation, Immune response and RNA splicing modules in breast cancer. Breast Cancer Res 2008, 10:R93.

7. Yu JX, Sieuwerts AM, Zhang Y, Martens JWM, Smid M, Klijn JGM, Wang Y, Foekens JA: Pathway analysis of gene signatures predicting metastasis of node-negative primary breast cancer. BMC Cancer 2007, 7:182.

8. Haibe-Kains B, Desmedt C, Sotiriou C, Bontempi G: A comparative study of survival models for breast cancer prognostication based on microrarray data: does a single gene beat them all? Bioinformatics 2008, 24:2200-2208

9. Lai C, Reinders MJT, van't Veer $\sqcup$, Wessels LFA: A comparison of univariate and multivariate gene selection techniques for classification of cancer datasets. BMC Bioinfo 2006, 7:235.

10. Segal E, Shapira M, Regev A, Pe'er D, Botstein D, Koller D, Friedman N: Module networks: identifying regulatory modules and their conditionspecific regulators from gene expression data. Nat Genet 2003, 34:166-176

11. Yousef M, Jung S, Showe LC, Showe MK: Recursive Cluster Elimination (RCE) for classification and feature selection from gene expression data. BMC Bioinfo 2007, 8:article 144.

12. van Vliet MH, Klijn CN, Wessels LFA, Reinders MJT: Module-Based Outcome Prediction Using Breast Cancer Compendia. PLOS ONE 2007 2:.

13. Chuang HY, Lee E, Liu YT, Lee D, Ideker T: Network-based classification of breast cancer metastasis. Mol Sys Biol 2007, 3:

14. Svensson JP, Stalpers $L J A$, van Lange REEE, Franken NAP, Haveman J, Klein B, Turesson I, Vrieling H, Giphart-Gassler M: Analysis of Gene Expression Using Gene Sets Discriminates Cancer Patients with and without Late Radiation Toxicity. PLoS Medicine 2006, 3:e422.

15. Ashburner M, Ball CA, Blake JA, Botstein D, an J M Cherry HB, Davis AP, Dolinski K, Dwight SS, Eppig JT, Harris MA, Hill DP, Issel-Tarver L, Kasarskis A, Lewis S, Matese JC, Richardson JE, Ringwald M, Rubin GM, Sherlock G:
Gene Ontology: tool for the unification of biology. Nat Genet 2000, 25:25-29.

16. Kim SY, Kim YS: A gene sets approach for identifying prognostic gene signatures for outcome prediction. BMC Genomics 2008, 9:

17. Lee E, Chuang HY, Kim JW, Ideker T, Lee D: Inferring Pathway Activity toward Precise Disease Classification. PLoS Comput Biol 2008, 4:

18. Guo Z, Zhang T, Li X, Wang Q, Xu J, Yu H, Zhu J, Wang H, Wang C, Topol EJ, Wang Q, Rao S: Towards precise classification of cancers based on robust gene functional expression profiles. BMC Bioinfo 2005, 6:article 58.

19. Bild AH, Yao G, Chang JT, Wang Q, Potti, Chasse D, Joshi MB, Harpole D, Lancaster JM, Berchuck A, Olson JA, Marks JR, Dressman HK, West M, Nevins JR: Oncogenic pathway signatures in human cancers as a guide to targeted therapies. Nature 2006, 439(7074):353-357.

20. Törönen $P$, Ojala PJ, Maartinen $P$, Holm L: Robust extraction of functional signals from gene set analysis using a generalized threshold free scoring function. BMC Bioinfo 2009, 10:307.

21. Subramanian A, Tamayo P, Mootha VK, Mukherjee S, Ebert BL, Gillette MA, Paulovich A, Pomeroy SL, Golub TR, Lander ES, Mesirov JP: Gene set enrichment analysis: A knowledge-based approach for interpreting genome-wide expression profiles. Proc Natl Acad Sci 2005, 102:15545-15550

22. Ackermann $M$, Strimmer $K$ : A general modular framework for gene set enrichment analysis. BMC Bioinfo 2009, 10:47.

23. Edgar R, Domrachev M, Lash AE: Gene Expression Omnibus: NCBI gene expression and hybridization array data repository. Nucl Acid Res 2002, 30:207-210

24. Wang Y, Klijn JG, Zhang Y, Sieuwerts AM, Look MP, Yang F, Talantov D, Timmermans M, van Gelder MM, Yu J, Jatkoe T, Berns EM, Atkins D, Foekens JA: Gene-expression profiles to predict distant metastasis of lymph-node-negative primary breast cancer. The Lancet 2005, 365:671-679.

25. Ivshina AV, George J, Senko O, Mow B, Putti TC, Smeds J, Lindahl T, Pawitan Y, Hall P, Nordgren H, Wong JE, Liu ET, Bergh J, Kuznetsov VA, Miller LD: Genetic reclassification of histologic grade delineates new clinical subtypes of breast cancer. Cancer Res 2006, 66:10292-10301.

26. Loi S, Haibe-Kains B, Desmedt C, Lallemand F, Tutt AM, Gillet C, Ellis P, Harris A, Bergh J, Foekens JA, Klijn JGM, Larsimont D, Buyse M, Bontempi G, Delorenzi M, Piccart MJ, Sotiriou C: Definition of Clinically Distinct Molecular Subtypes in Estrogen Receptor-Positive Breast Carcinomas Through Genomic Grade. J Clin Oncol 2007, 25:1239-1246.

27. Loi S, Haibe-Kains B, Desmedt C, Wirapati P, Lallemand F, Tutt AM, Gillet C, Ellis P, Ryder K, Reid JF, Daidone MG, Pierotti MA, Berns EM, Jansen MP, Foekens JA, Delorenzi M, Bontempi G, Piccart MJ, Sotiriou C: Predicting prognosis using molecular profiling in estrogen receptor-positive breast cancer treated with tamoxifen. BMC Genomics 2008, 9:

28. Desmedt C, Piette F, Loi S, Wang Y, Lallemand F, Haibe-Kains B, Viale G, Delorenzi M, Zhang Y, d'Assignies MS, Bergh J, Lidereau R, Ellis P, Harris AL, Klijn JG, Foekens JA, Cardoso F, Piccart MJ, Buyse M, Sotiriou C, Consortium T: Strong time dependence of the 76-gene prognostic signature for node-negative breast cancer patients in the TRANSBIG multicenter independent validation series. Clin Cancer Res 2007, 13:3207-3214

29. Schmidt M, Böhm D, von Törne C, Steiner E, Puhl A, Pilch H, Lehr HA, Hengstler JG, Kölbl J, Gehrmann M: The Humoral Immune System Has a Key Prognostic Impact in Node-Negative Breast Cancer. Cancer Res 2008, 68:5405-5413.

30. Harrell FE: Regression Modeling Strategies Springer; 2001.

31. Kanehisa M, Goto S: KEGG: Kyoto Encyclopedia of Genes and Genomes. Nucl Acid Res 2000, 28:27-30

32. Tian L, Greenberg SA, Kong SW, Altschuler J, Kohane IS, Park PJ: Discovering statistically significant pathways in expression profiling studies. Proc Natl Acad Sci 2005, 102:13544-13549.

33. Efron B, Tibshirani R: On testing the significance of sets of genes. Annal Stat 2007, 1:107-129.

34. Park MY, Hastie T, Tibshirani R: Averaged gene expressions for regression. Biostatistics 2007, 8:212-227.

35. Lehmann EL: Nonparametrics. Statistical Methods Based on Ranks McGrawHill; 1975.

36. Goeman JJ, Bühlmann P: Analyzing gene expression data in terms of gene sets: methodological issues. Bioinformatics 2007, 23:980-987. 
37. Barry WT, Nobel AB, Wright FA: A statistical framework for testing functional categories in microarray data. Ann Appl Stat 2008, 2:286-315.

38. Schölkopf B, Smola AJ: Learning with Kernels MIT Press; 2002.

39. Bedo J, Sanderson C, Kowalczyk A: An Efficient Alternative to SVM Based Recursive Feature Elimination with Applications in Natural Language Processing and Bioinformatics. Proc Aust Joint Conf Al 2006.

40. Tibshirani R, Hastie T, Narasimhan B, Chu G: Class Prediction by Nearest Shrunken Centroids, with Applications to DNA Microarrays. Stat Sci 2003, 18:104-117.

41. Dabney AR, Storey JD: Optimality driven nearest centroid classification from genomic data. PLoS One 2007, 2:e1002.

42. McLachlan GJ, Do KA, Ambroise C: Analyzing Microarray Gene Expression Data Wiley Interscience; 2004

43. Varma S, Simon R: Bias in error estimation when using cross-validation for model selection. BMC Bioinfo 2006, 7:

44. Binder H, Schumacher M: Adapting Prediction Error Estimates for Biased Complexity Selection in High-Dimensional Bootstrap Samples. Statist Appl Genet Mol Biol 2008, 7:

45. Hastie T, Tibshirani R, Friedman J: The Elements of Statistical Learning Springer; 2001.

46. Downward J: Targeting RAS signalling pathways in cancer therapy. Nat Rev Cancer 2003, 3:11-22.

47. Dai H, van't Veer L, Lamb J, He YD, Mao M, Fine BM, Bernards R, Vijver M van de, Deutsch P, Sachs A, Stoughton R, Friend S: A cell proliferation signature is a marker of extremely poor outcome in a subpopulation of breast cancer patients. Cancer Res 2005, 65:4059-4066.

48. Mosley JD, Keri RA: Cell cycle correlated genes dictate the prognostic power of breast cancer gene lists. BMC Med Genom 2008, 1:11.

49. van Diest PJ, Wall E van der, Baak JPA: Prognostic value of proliferation in invasive breast cancer: a review. J Clin Pathol 2004, 57:675-681.

50. Sørlie T, Tibshirani R, Parker J, Hastie T, Marron JS, Nobel A, Deng S, Johnsen H, Pesich R, Geisler S, Demeter J, Perou CM, Lønning PE, Brown $\mathrm{PO}$, Børresen-Dale AL, Botstein D: Repeated observation of breast tumor subtypes in independent gene expression data sets. Proc Nat/ Acad Sci 2003, 100:8418-8423.

51. Desmedt C, Haibe-Kains B, Wirapati P, Buyse M, Larsimont D, Bontempi G, Delorenzi M, Piccart M, Sotiriou C: Biological processes associated with breast cancer clinical outcome depend on the molecular subtypes. Clin Cancer Res 2008, 14:5158-5165.

52. Buyse M, Loi S, van 't Veer L, Viale G, Delorenzi M, Glas AM, d'Assignies MS, Bergh J, Lidereau R, Ellis P, Harris A, Bogaerts J, Therasse P, Floore A, Amakrane M, Piette F, Rutgers E, Sotiriou C, Cardoso F, Piccart MJ, Consortium T: Validation and clinical utility of a 70-gene prognostic signature for women with node-negative breast cancer. JNCl 2006 , 98:1183-1192

53. Dent R, Trudeau M, Pritchard KI, Hanna WM, Kahn HK, Sawka CA, Lickley LA, Rawlinson E, Sun P, Narod SA: Triple-negative breast cancer: clinical features and patterns of recurrence. Clin Cancer Res 2007, 13:4429-4434.

54. Teschendorff AE, Naderi A, Barbosa-Morais NL, Pinder SE, Ellis IO, Aparicio S, Brenton JD, Caldas C: A consensus prognostic gene expression classifier for ER positive breast cancer. Genome Biol 2006, 7:R101.

55. Goeman J: penalized. L1 (lasso) and L2 (ridge) penalized estimation in GLMs and in the Cox model 2008 [http://www.msbi.nl/goeman]. [R package version 0.9-22]

56. Karatzoglou A, Smola A, Hornik K, Zeileis A: kernlab - An S4 Package for Kernel Methods in R. Journal of Statistical Software 2004, 11(91-20 [http:/ /www.jstatsoft.org/v11/i09/

57. Morley M, Molony CM, Weber TM, Devlin JL, Ewens KG, Spielman RS, Cheung VG: Genetic analysis of genome-wide variation in human gene expression. Nature 2004, 430:743-747.

58. Brentani H, Caballero OL, Camargo AA, da Silva AM, da Silva WA, Neto ED, Grivet M, Gruber A, Guimaraes PEM, Hide W, Iseli C, Jongeneel CV, Kelso J, Nagai MA, Ojopi EPB, et al.: The generation and utilization of a canceroriented representation of the human transcriptome by using expressed sequence tags. Proc Natl Acad Sci 2003, 100:13148-13423.

doi: 10.1186/1471-2105-11-277

Cite this article as: Abraham et al., Prediction of breast cancer prognosis using gene set statistics provides signature stability and biological context BMC Bioinformatics 2010, 11:277

Submit your next manuscript to BioMed Central and take full advantage of:

- Convenient online submission

- Thorough peer review

- No space constraints or color figure charges

- Immediate publication on acceptance

- Inclusion in PubMed, CAS, Scopus and Google Scholar

- Research which is freely available for redistribution 